Mohammed Al-Zayer, BDS, ${ }^{1}$ Marcio da Fonseca, DDS, MS, ${ }^{1 *}$ Jonathan A. Ship, $\mathrm{DMD}^{2}$

${ }^{i}$ Department of Orthodontics and Pediatric Dentistry, University of Michigan School of Dentistry, 1011 N. University Ave., Ann Arbor, MI 48109; and ${ }^{2}$ Division of Biological Sciences, Medicine, Surgery, New York University College of Dentistry; *corresponding author, marcio@umich.edu

Spec Care Dentist 21(5):187-190, 2001

\section{Pyogenic granuloma in a renal transplant patient: case report}

\section{INTRODUCTION}

D yogenic granuloma is a well-circumscribed, benign soft-tissue growth of an inflammatory nature arising from the connective tissue of the skin or mucous membrane. ${ }^{1-5}$ It may present as an ulcerated surface which can be necrotic and covered by a white slough resembling pus, hence the name. ${ }^{6,7}$ It occurs anywhere in the body, including the fingers and toes. ${ }^{8}$ The most commonly affected intra-oral site is the gingiva, especially the anterior maxillary area. In descending order of frequency, the lesion may arise on the lips, tongue, buccal mucosa, palate, and alveolar mucosa of edentulous areas. ${ }^{1,2,4,5,8,9}$ Clinically it appears as a smooth or lobulated, pedunculated, or sessile mass. ${ }^{1,6,9-11}$ Its color ranges from pink to red to purple, with a gray pseudomembrane over the surface secondary to ulceration of the overlying epithelium. ${ }^{1,3,5,6,8,9}$ It is highly vascular and can bleed upon manipulation or spontaneously., ${ }^{4,8-11}$ If hemorrhage occurs inside the lesion, it becomes melanotic brown. ${ }^{5}$ During its early stages, it is soft or spongy in consistency, and as it ages, the capillaries can become obliterated, leading to a more fibrous appearance. ${ }^{1,2,5,7,9}$ The lesions are usually painless, but on rare occasions, patients complain of a dull, well-localized pain in the area of the tumor. ${ }^{1,4,9-11}$ Pyogenic granulomas grow very rapidly, which may at first suggest a malignant lesion to the dentist. After reaching a size varying from a few millimeters to several centimeters, the lesion remains static. ${ }^{1,2,5,8,9}$ In large, long-standing lesions, there may be radiographic evidence of localized alveolar bone resorption. ${ }^{1,2,6}$ Both sexes are affected, but there is a higher incidence seen in females. ${ }^{1,2,9,10}$ The granuloma may occur at any age, but it has been reported that $60 \%$ of the lesions are seen in persons between 11 and 40 years of age. ${ }^{1,4,5}$ Some studies showed a higher prevalence in Caucasians, but most authors believe that these lesions have no racial predilection. $1,4,5$

This paper describes the case history of an adolescent female who had had a renal transplant and was referred to our pediatric dental service because of drug-induced gingival overgrowth. Examination of the anterior maxillary area showed a red, vascular, exophytic mass which had been excised a few months earlier and grew rapidly. No biopsy was done at the time of the initial surgery.

\section{CASE REPORT}

A 14-year-old female was referred to our pediatric dental clinic by the Nephrology service for evaluation and treatment of cyclosporine-induced gingival hyperplasia. Her past medical history was significant for nephrotic syndrome, renal transplantation, juvenile fibroadenomas of the breast, and allergy to codeine. Her current medications included cyclosporine, prednisone, hydrochlorothiazide, omeprazole, and atorvastatin. The patient's main complaint was the gingival enlargement in the maxillary anterior region (Fig 1) that bled at times and interfered with eating and oral care. In addition, it was esthetically unpleasant, and she wanted it removed. 


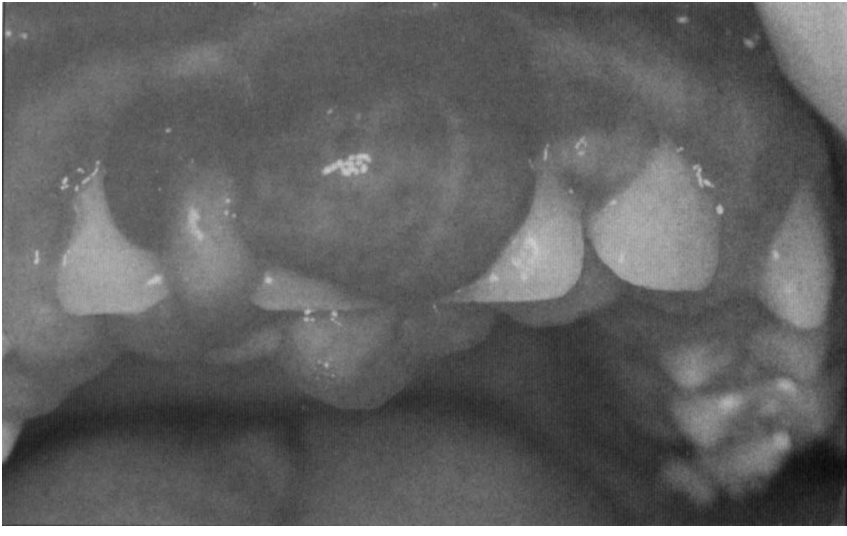

Figure 1. Exophytic, pedunculated, vascular lesion in the anterior maxillary area.

The extra-oral examination was within normal limits except for mild hirsutism. The patient had her permanent dentition, had several restorations, and was caries-free. Although an examination of a maxillary occlusal radiograph (Fig 2) revealed mild bone loss, there was no tooth mobility or displacement. The intra-oral soft-tissue examination revealed fair oral hygiene and generalized mild gingival overgrowth consistent with long-term use of cyclosporine (Fig 3), with the exception of the maxillary anterior area (Fig 1), where a red, exophytic, pedunculated vascular growth covered the central incisors and extended into the palate. In August, 1999, the patient was examined by a periodontist who surgically excised a similar

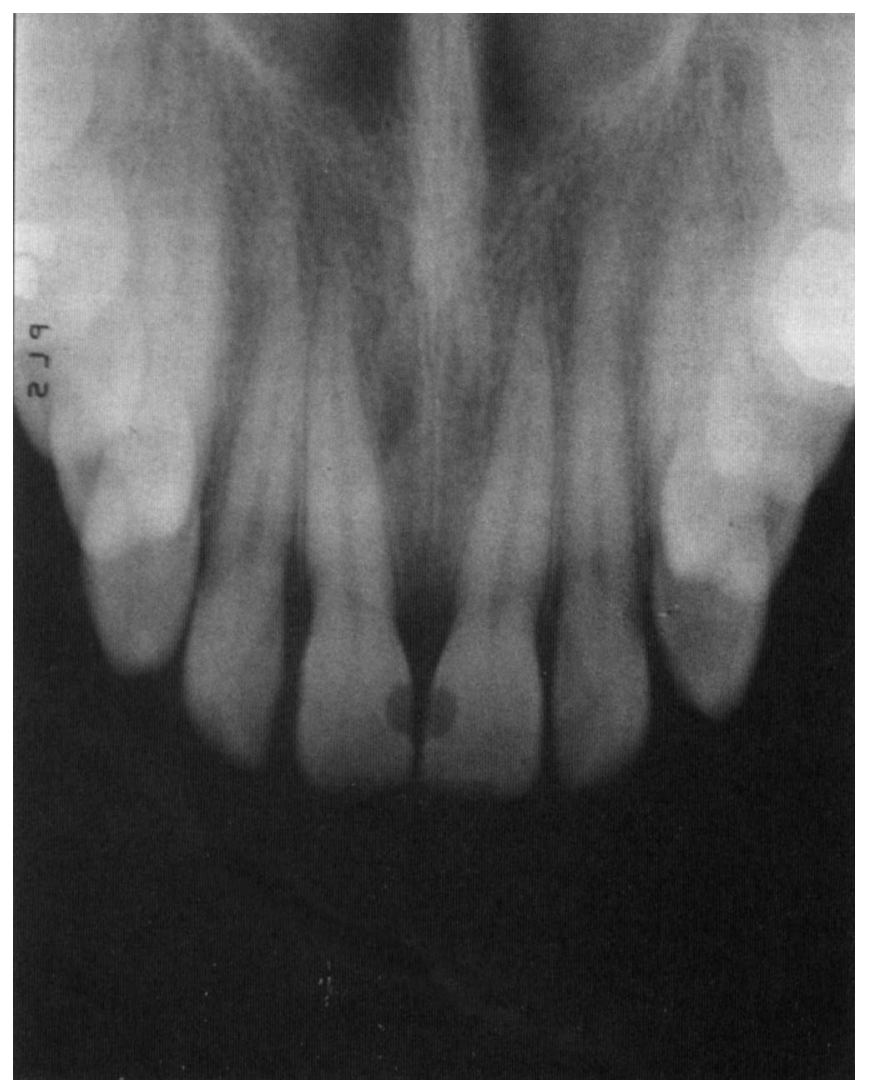

Figure 2. Bone loss in the anterior maxillary area. mass that was present over the facial surface of the incisors. A biopsy was not done at that time, and the tissue grew back to its previous size over a two-month period. Upon consultation with the Oral Medicine service and consent from the patient and her mother, an excisional biopsy (Fig 4) of the maxillary anterior area was performed under local anesthesia and sent to the Oral Pathology laboratory for a definitive diagnosis. The histological examination reported that the lesion was partially covered by regenerating epithelium, which presented an ulcer with a mixed inflammatory infiltrate. Beneath the necrotic surface, granulation tissue was evident with fibrovascular connective tissue and large congested capillaries, surrounded by a mixed chronic inflammatory infiltrate. A diagnosis of pyogenic granuloma was made. The patient returned a few weeks later for a gingivectomy and complete excision of the granuloma. No complications occurred, and the patient will return for follow-up every 3 months in the first year after surgery and every 6 months thereafter.

\section{DISCUSSION}

Many individuals who take cyclosporine on a long-term basis, like our patient, are at risk of developing gingival overgrowth which is generally limited to the keratinized gingiva. As seen in Fig 3 , the tissue in the mandibular anterior area appeared firm, with focal lobulations, having a stippled and pink surface, ${ }^{12}$ a strikingly different appearance from the maxilla (Fig 1). This lesion was remarkable in that although it had been removed less than 8 months previously, it grew to its previous size in just a few weeks. Concerns arose about a possible malignancy because of the lesion's rapid growth and an increased incidence of cancer in patients who have been on prolonged or intensive immunosuppressive therapy associated with organ transplantation. The most common cancers are skin cancers, non-Hodgkin's lymphoma, Kaposi's sarcoma, hepatobiliary carcinomas, and carcinomas of the uterine cervix, the vulva, and the perineum. ${ }^{13,14}$ Therefore, a biopsy of the tissue was deemed necessary for the appropriate course of treatment to be implemented. The histopathological examination reported a pyogenic granuloma.

The occurrence of oral pyogenic granulomas depends on several factors, such as the presence of a sufficient amount of connective tissue, its degree of inflammatory responsiveness to various causes, the degree of vulnerability of the area to trauma and irritation, the presence of teeth or prostheses, and the oral hygiene of the patient. ${ }^{1}$ The floor of the mouth is possibly the only region that is spared because of the small amount of connective tissue and the action of the tongue protecting against traumatic and irritating factors. ${ }^{1}$ The anterior maxilla and the facial gingiva are most often affected. $1,2,4,11$

Although the exact etiology is unknown, pyogenic granulomas have been classically viewed as hyperplastic granulation tissue that reacts to local irritation (calculus, prostheses, overhanging edges or rough restorative surfaces), trauma, poor oral hygiene, toothbrushing microtrauma, and hormonal changes. ${ }^{1-5,7,9-11,15}$ Some authors ${ }^{8}$ consider them unrelated to gingivitis and periodontitis, although an adjacent gingival inflammation may be evident, as seen in this subject (Fig 1). Pyogenic granulomas can occur after dental extractions, especially third molars, possibly in response to irritants that have fallen into the sockets, such as calculus, food, tooth fragments, and bony spicules. ${ }^{8}$ They are common during 


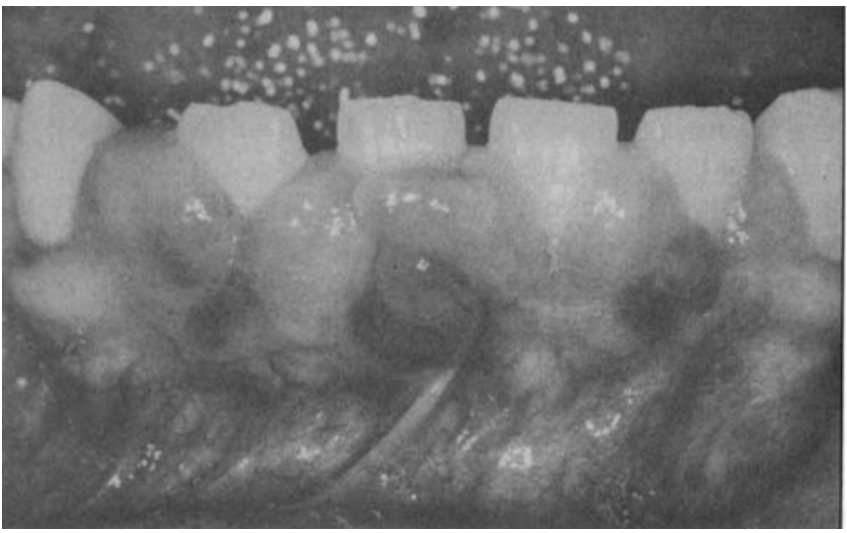

Figure 3. Lobulated, stippled, firm gingiva in the lower anterior area consistent with long-term use of cyclosporine.

pregnancy ("pregnancy tumor," "granuloma gravidarum"), particularly during the second and third trimesters, and tend to involute after delivery. $2,5,8,9,11$ Because of the neutrophils present in the lesion, many authors believed that oral micro-organisms were a triggering factor for the granuloma, but that hypothesis has been dismissed. ${ }^{4,5,8,9}$ Although most researchers consider hormonal changes as an etiological factor, others disagree because of their low recurrence rate and almost identical gender distribution. ${ }^{4,5}$ However, a clear female predilection $^{1,2,9,10}$ and high recurrence ${ }^{3}$ have been reported.

Another possible factor in the development of these lesions is the chronic use of cyclosporine. Some researchers, describing non-gingival soft-tissue growths in bone marrow transplant recipients who were receiving the drug for graft-vs.-host disease prevention, stated that it is possible that cyclosporine triggered and exaggerated the proliferative response of the connective tissue, leading to the development of the soft-tissue masses. ${ }^{15,16}$ Therefore, one is tempted to postulate that cyclosporine has a role in the etiology of our patient's lesion. Furthermore, Yuan et al. ${ }^{17}$ reported that five polypeptides (Tie-2, angiopoietin-1, angiopoietin-2, ephrin-B2, and ephrin-B4) may play an important role in the process of adult inflammatory neovascularization, particularly in pyogenic granuloma. These authors also claim that the etiology of pyogenic granulomas could be due to an imbalance between angiogenesis enhancers and inhibitors. ${ }^{18}$

The definitive diagnosis can be made only by histopathologic examination, 1,6 which shows granulation tissue, with anastomosing endothelial-lined vascular spaces with fibroblast proliferation and endothelial cell budding. $2,5,6,8,9,11$ The overlying stratified squamous epithelium is often thin and atrophic and may be partially ulcerated, presenting a fibrinous exudate with entrapped leukocytes. ${ }^{5,6,8,9}$ Neutrophils, plasma cells, and leukocytes infiltrate the loose connective tissue dispersed throughout the fibrovascular tissue. ${ }^{6,8,9,11}$ The differential diagnosis should include peripheral giant cell granuloma, capillary hemangioma, metastatic tumor, Kaposi's sarcoma, angiosarcoma, malignant melanoma, parulis, peripheral ossifying fibroma, peripheral fibroma, leiomyoma, hemangioendothelioma, hemangiopericytoma, cytomegalovirus infection, and bacillary angiomatosis. $^{5,6,11}$ In a review of 834 consecutive biopsies of gingival lesions, pyogenic granulomas were the most common lesion $(23.6 \%){ }^{19}$

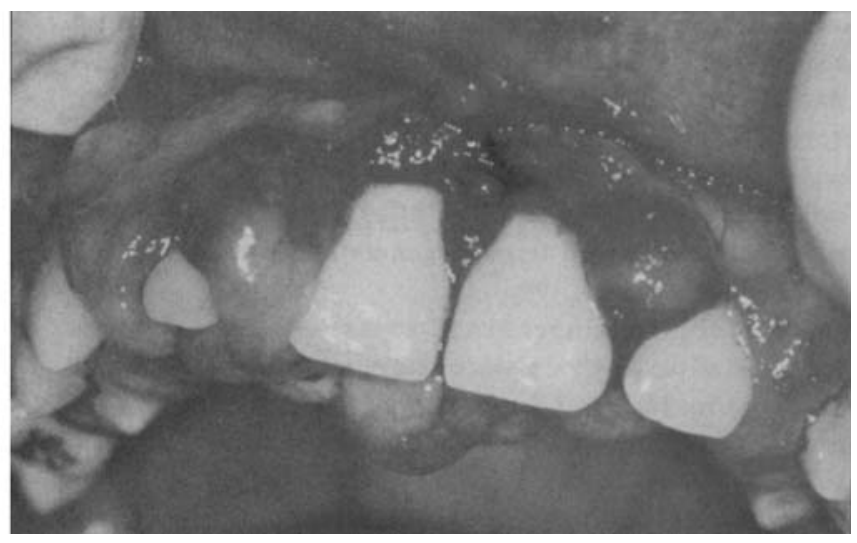

Figure 4. Anterior maxillary area after biopsy.

Treatment consists of surgical excision. It has been reported that if excision is done to include the base of the mass, the lesion will not recur. ${ }^{4-10,15}$ In the case history presented here, it is likely that the recurrence was due to only partial removal of the lesion. Scaling and curettage of adjacent teeth and root surfaces are recommended, because calculus is a local irritant that can trigger recurrence. ${ }^{8,9,11}$ As seen in this patient, there may be localized bone loss associated with the lesion (Fig 2). Occlusal and/or panoramic radiographs are of importance for the evaluation of alveolar bone resorption, which could lead to tooth loss. In lesions that are very large or deeply embedded in the palate, the dentist should, as part of informed consent, discuss with the patient and the caretakers the risk of transient or permanent palatal numbness if the nasopalatine nerve is damaged during the surgical procedure.

In summary, dental professionals should be aware that, in patients who take medications which can induce gingival overgrowth (cyclosporine, phenytoin, nifedipine), a biopsy is indicated for all gingival lesions. As Layfield et al. ${ }^{20}$ remarked, after a diagnostic survey of thousands of gingival biopsies, knowledge of the frequency and presentation of the most common lesions found in the gingiva is very beneficial in dental practice.

\section{REFERENCES}

1. Angelopoulos AP. Pyogenic granuloma of the oral cavity: statistical analysis of its clinical features. J Oral Surg 29:840-47, 1971.

2. Leyden JJ, Master GH. Oral cavity pyogenic granuloma. Arch Dermatol 108:226-28, 1973.

3. Vilmann A, Vilmann P, Vilmann H. Pyogenic granuloma: evaluation of oral conditions. Br J Oral Maxillofac Surg 24:376-82, 1986.

4. Bhaskar SN, Jacoway JR. Pyogenic granuloma-clinical features, incidence, histology, and result of treatment: report of 242 cases. $J$ Oral Surg 24:391-98, 1966.

5. Kerr DA. Granuloma pyogenicum. J Oral Surg 4:158-76, 1951.

6. Goodman-Topper ED, Bimstein E. Pyogenic granuloma as a cause of bone loss in a twelve year-old child: report of case. $J$ Dent Child 61:65-7, 1994.

7. Mody RN, Raut D. Large pyogenic granuloma: a case report. Dent Update 23:412-3, 1996.

8. Sapp JP, Eversole LR, Wysocki GP. Contemporary oral and maxillofacial pathology. St. Louis: Mosby, pp. 305-7, 1997. 
9. Neville BW, Damm DD, Allen CM, et al. Oral and maxillofacial pathology. Philadelphia: W.B. Saunders, pp. 371- 3, 1995.

10. Lawoyin JO, Arotiba JT, Dosumu OO. Oral pyogenic granuloma: a review of 38 cases from Ibadan, Nigeria. $\mathrm{Br} J$ Oral Maxillofac Surg 35:185-9, 1997.

11. Fowler EB, Cuenin MF, Thompson SH, et al. Pyogenic granuloma associated with guided tissue regeneration: a case report. $J$ Periodontol 67:1011-15, 1996.

12. Wysocki GP, Gretzinger HA, Laupacis A, et al. Fibrous hyperplasia of the gingiva: a side effect of cyclosporine A therapy. Oral Surg Oral Med Oral Pathol 55:274-8, 1983.

13. Penn I. Tumors after renal and cardiac transplantation. Hematol Oncol Clin N Am 7:431-45, 1993.

14. DiGiovanna JJ. Posttransplantation skin cancer: scope of the problem, management, and role of systemic retinoid chemoprevention. Transplant Proc 30:2771-8, 1998.

15. Lee L, Miller PA, Maxymiw WG, et al. Intraoral pyogenic granuloma after allogeneic bone marrow transplant - report of three cases. Oral Surg Oral Med Oral Pathol 78:607-10, 1994.

16. Woo SB, Allen CM, Orden A, et al. Nongingival soft tissue growths after allogeneic marrow transplantation. Bone Marrow Transplant 17:1127-32, 1996.

17. Yuan K, Jin YT, Lin MT. Expression of Tie-2, angiopectin-1, angiopectin-2, ephrinB2 and EphB4 in pyogenic granuloma of human gingiva implicates their roles in inflammatory angiogenesis. J Periodontal Res 35:165-71, 2000.

18. Yuan $\mathrm{K}$, Jin YT, Lin MT. The detection and comparison of angiogenesis-associated factors in pyogenic granuloma by immunohistochemistry. J Periodontol 71:701-9, 2000.

19. Stablein MJ, Silverglade LB. Comparative analysis of biopsy specimens from gingiva and alveolar mucosa. $J$ Periodontol 56:671-6, 1985.

20. Layfield LL, Shopper TO, Weir JC. A diagnostic survey of biopsied gingival lesions. J Dent Hyg 69:175-9, 1995. 